\title{
Modelling of confinement degradation in the radiative improved mode caused by a strong gas puff
}

\author{
D Kalupin ${ }^{1}$, M Z Tokar ${ }^{2}$, P Dumortier ${ }^{1}$, A Messiaen ${ }^{1}$, D Reiser ${ }^{2}$, \\ S Soldatov $^{3}$, B Unterberg ${ }^{2}$, G van Wassenhove ${ }^{1}$ and R Weynants ${ }^{1}$ \\ ${ }^{1}$ Laboratoire de Physique des Plasmas / Laboratorium voor Plasmafysica, EURATOM \\ Association, Ecole Royale Militaire, B-1000 Brussels, Belgium \\ ${ }^{2}$ Institut für Plasmaphysik, Forschungszentrum Jülich GmbH, EURATOM Association, D-52425 \\ Jülich, Germany \\ ${ }^{3}$ Nuclear Fusion Institute, Russian Research Centre 'Kurchatov Institute', Kurchatov Square 1, \\ 123182 Moscow, Russia
}

Received 12 March 2001

\begin{abstract}
Confinement deterioration and rollover from the radiative improved (RI) mode to the low (L) confinement mode in plasmas with a strong gas puff in the tokamak TEXTOR-94 are modelled by the one-dimensional transport code RITM (radiation from impurities in the transport model). The anomalous transport coefficients in the plasma core include contributions from the ion temperature gradient and dissipative trapped electron instabilities. This model allows us to reproduce the L-RI bifurcation with impurity seeding in good agreement with experimental observations. Whereas the transport at the plasma edge under the RI-mode conditions might be described by the electrostatic turbulence caused by electric currents in the scrape-off layer of the limiter, the present computations show that the level of the edge transport must be increased by at least a factor of three in order to reproduce the evolution of the plasma density profile and the effective ion charge during RI-to-L back transitions. An increase of this order is in agreement with reflectometer measurements and could tentatively be explained by the effect of neutrals on resistive drift modes.
\end{abstract}

\section{Introduction}

Gas puffing is the simplest and most frequent method for controlling the plasma density in fusion devices. However, as has been found in many tokamaks, a puffing of too much intensity can lead to a progressive deterioration of the energy and particle confinement. In particular, a back transition from the high $(\mathrm{H})$ confinement mode to the low (L) confinement mode has been observed in the divertor tokamaks, JET, JT-60, DIII-D and ASDEX-U (Horton et al 1999, Petrie et al 1993, Suttrop et al 1999) during a strong gas puff. Normally, this development is accompanied by a decrease of the averaged density time derivative, $\partial \bar{n} / \partial t$, and finally can lead to the fuelling density limit (Greenwald et al 1988). 
Experiments with different scenarios of gas puff have recently been performed on the TEXTOR-94 tokamak in discharges where seeding of neon triggered a transition from the L-mode to the radiative improved (RI) mode (Mank et al 2000, Unterberg et al 2000). These experiments were aimed at attaining plasma densities significantly exceeding the Greenwald limit, $n_{\mathrm{Gr}}\left(10^{20} \mathrm{~m}^{-3}\right)=I_{\mathrm{P}} / \pi a^{2}$, where $I_{\mathrm{P}}$ is the plasma current (MA) and $a$ is the minor radius $(\mathrm{m})$, with simultaneous sustaining of the improved confinement. It has been found that a strong gas puff results in a moderate increase of the line-averaged density characterized by a flattening of the density profile in which the edge plasma density rises significantly above the averaged density. At the same time the content of neon particles in the plasma volume was drastically reduced such that the effective plasma charge, $Z_{\text {eff }}$, was decreased to its L-mode level. The energy content in the plasma was diminished and a rollover to the L-mode occurred (see figure 1, solid curves).

Conversely, a less intensive gas puff allowed us to preserve the energy confinement of the H-mode, or obtain even higher quality, and to maintain a peaked density profile, which is of principal importance for RI-mode performance. Simultaneously, the averaged density was increased noticeably to a level exceeding $n_{\mathrm{Gr}}$ by $30 \%$ (see figure 1 , dashed curves).

In this paper, we report on the results of transport modelling performed in order to interpret these and other experiments with gas puffing into the RI-mode in TEXTOR-94. The modelling tool, the one-dimensional (1D) transport code RITM (radiation from impurities in the transport model) and the semi-empirical anomalous transport model used in the present simulations of

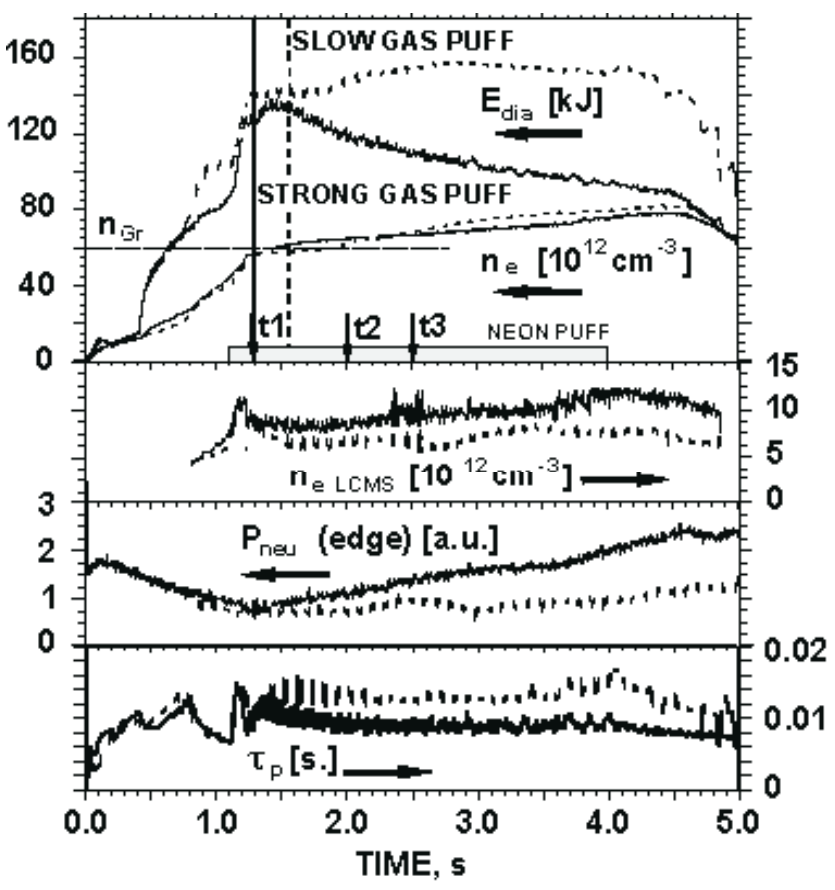

Figure 1. Two discharges differing by the gas puff rate: 88478 (dashed curves), slow gas puff$3 \times 10^{21}$ part s ${ }^{-1} ; 88482$ (solid curves), strong gas puff $-8 \times 10^{21}$ part s$^{-1}$. $E_{\text {dia }}$ is the diamagnetic energy, $n_{\mathrm{e}}$ is the line-averaged electron density, $n_{\mathrm{e}}$ (LCMS) is the electron density at the last closed magnetic surface, $P_{\text {neu }}$ is the neutral pressure at the edge, $\tau_{\mathrm{p}}$ is the particle confinement time, $n_{\mathrm{Gr}}$ indicates the Greenwald density limit, and $t 1, t 2$ and $t 3$ are the times of the reflectometer measurements. 
L- and RI-modes, will be discussed in section 2. The calculations given in section 3 show that the experimentally observed modifications in plasma density and in impurity content can only be simulated if one assumes a strong increase of anomalous particle diffusion at the very plasma edge during intense gas puffing. This conclusion is supported by reflectometer measurements of the density fluctuation spectra, which will be presented in section 4. Finally, we discuss a mechanism of the influence of puffed neutrals on anomalous transport, which provides a possible explanation for our findings.

\section{Transport code and model}

\subsection{The RITM code, transport equations}

In order to model the plasma profiles in both the L- and RI-modes in TEXTOR, the 1D transport code RITM (Tokar 1994) has been used. This code was developed to simulate, in a self-consistent manner, the transport and influence of impurities in diverse plasma configurations. RITM firstly solves in a diffusive approximation the kinetic equations for the velocity distribution functions $f_{j}\left(v_{r}\right)$ of neutral particles:

$$
\frac{1}{r g_{1}} \frac{\partial}{\partial r}\left(r g_{1} v_{r} f_{j}\right)=S_{j}-v_{j} f_{j}
$$

where $r$ is the minor radius of the magnetic surface, $j=1-4$ corresponds to neutral molecules and reflected atoms generated at the limiter, Franck-Condon and hot atoms arising in the plasma in processes of ionization, dissociation and charge-exchange, and $S_{j}$ and $v_{j}$ are the sources and the frequencies of disintegrative processes for the corresponding kinds of neutrals. The Shafranov shift computed from the Grad-Shafranov equation in cylindrical approximation is used with analytically prescribed elongation and triangularity of the magnetic surfaces to determine the metric coefficients $g_{1,2}$ which differ from 1 due to toroidicity (Solov'ev and Shafranov 1970).

The transport of charged particles is described by the continuity equations for electrons

$$
\frac{\partial n_{\mathrm{e}}}{\partial t}+\frac{1}{r g_{1}} \frac{\partial}{\partial r}\left(r g_{2} \Gamma_{\perp}^{\mathrm{e}}\right)=S_{\mathrm{n}}+\sum_{Z} Z S_{Z}
$$

and impurity ions of all charge states of $\mathrm{He}, \mathrm{C}, \mathrm{O}, \mathrm{Ne}$ and $\mathrm{Si}$

$$
\frac{\partial n_{Z}}{\partial t}+\frac{1}{r g_{1}} \frac{\partial}{\partial r}\left(r g_{2} \Gamma_{\perp}^{Z}\right)=S_{Z}
$$

with $n_{\mathrm{e}, Z}$ being the densities of the electrons and impurity species of the charge $Z$. Both diffusive and convective contributions are included in the particle flux densities

$$
\Gamma_{\perp}^{\mathrm{e}}=-D_{\perp} \frac{\partial n_{\mathrm{e}}}{\partial r}+V_{\perp} n_{\mathrm{e}}, \quad \Gamma_{\perp}^{Z}=-D_{\perp}^{Z} \frac{\partial n_{Z}}{\partial r}+V_{\perp}^{Z} n_{Z}
$$

$S_{\mathrm{n}}$ is the net electron source due to ionization of hydrogen neutrals and $S_{Z}$ is the source of the impurity species. The density and flux of the background ions are computed from the quasi-neutrality conditions

$$
\begin{aligned}
& n_{\mathrm{i}}=n_{\mathrm{e}}-\Sigma Z \cdot n_{Z} \\
& \Gamma_{\perp}^{\mathrm{i}}=\Gamma_{\perp}^{\mathrm{e}}-\Sigma Z \cdot \Gamma_{\perp}^{Z} .
\end{aligned}
$$

Heat transport is governed by the following equations for the electron and ion temperatures, $T_{\mathrm{e}}$ and $T_{\mathrm{i}}$, and the latter is assumed to be the same for all ion species

$\frac{3}{2} \frac{\partial n_{\mathrm{e}} T_{\mathrm{e}}}{\partial t}+\frac{1}{r g_{1}} \frac{\partial}{\partial r}\left[r g_{2}\left(1.5 \Gamma_{\perp}^{\mathrm{e}} T_{\mathrm{e}}-\kappa_{\perp}^{\mathrm{e}} \frac{\partial T_{\mathrm{e}}}{\partial r}\right)\right]=\frac{J^{2}}{\sigma}+Q_{\mathrm{au}}^{\mathrm{e}}-Q_{\mathrm{ei}}-Q_{\mathrm{en}}-Q_{\mathrm{eI}}$ 


$$
\frac{3}{2} \frac{\partial n_{\Sigma} T_{\mathrm{i}}}{\partial t}+\frac{1}{r g_{1}} \frac{\partial}{\partial r}\left[r g_{2}\left(1.5 \Gamma_{\perp}^{\Sigma} T_{\mathrm{i}}-\kappa_{\perp}^{\Sigma} \frac{\partial T_{\mathrm{i}}}{\partial r}\right)\right]=Q_{\mathrm{au}}^{\mathrm{i}}-Q_{\mathrm{ei}}-Q_{\mathrm{in}}
$$

where $n_{\Sigma}=n_{\mathrm{i}}+\Sigma n_{Z}, \Gamma_{\Sigma}=\Gamma_{\mathrm{i}}+\Sigma \Gamma_{Z}, Q_{\mathrm{au}}^{\mathrm{e}, \mathrm{i}}$ are the densities of electron and ion heat sources due to additional heating from neutral beam injection (NBI) and ion cyclotron resonance heating (ICRH) estimated from TRANSP computations, $Q_{\text {ei }}, Q_{\text {en }}$ and $Q_{\text {eI }}$ are the energy losses from electrons due to Coulomb collisions with ions, excitation and ionization of neutrals and impurities, respectively, and $Q_{\text {in }}$ is the energy exchange between main ions and neutrals.

The time evolution of the current density profile $J(r)$ including both ohmic and bootstrap contributions is computed from the current diffusion equation with the conductivity $\sigma$ taking into account neoclassical corrections (Hirshman et al 1977).

The boundary conditions to the transport equations at the last closed magnetic surfaces (LCMS) are given by the e-folding lengths for the densities and temperatures taken from measurements. This, of course, limits the self-consistency of our model. A future development is planned to improve this by coupling with a description of the scrape-off layer (SOL) analogously to the approach realized by Baelmans et al (1995). Additionally, the total toroidal plasma current and the time evolution of the averaged plasma density are given. The latter is generated by choosing for each time step an appropriate influx of cold molecules in an iterative procedure. During the time interval of a strong puff the gas inflow is prescribed. The program for the neon puffing is chosen to reproduce the experimental evolution of the integral plasma radiation.

\subsection{Specific transport model}

Semi-empiric transport coefficients, which allow us to analytically reproduce the L-RI bifurcation brought about by the seeding of a sufficient amount of impurities (Tokar et al 2000), have recently been incorporated into RITM. In addition to neoclassical transport, it takes into account contributions to the anomalous transport of particles and energy from three types of instability: ion temperature gradient (ITG), dissipative trapped electron (DTE) and that caused by electric currents in the SOL (the corresponding contributions to the transport characteristics are identified by the superscript EDGE). Characteristic transport coefficients are estimated in the mixing length limit, $\chi_{\perp} \propto \gamma_{\max } / k_{\perp \text { max }}^{2}$ (Kadomtsev and Pogutse 1971), where $\gamma_{\max }$ is the maximum growth rate of the relevant unstable mode and $k_{\perp \text {,max }}$ is the perpendicular wavenumber at which this maximum is achieved. For $\gamma_{\max }$ the following expressions have been used (Nordman et al 1990, Tokar et al 2000, Kadomtsev and Pogutse 1971)

$\gamma_{\max }^{\mathrm{ITG}} \approx \frac{c T_{\mathrm{e}} k_{\perp, \text { max }}^{\mathrm{ITG}}}{e B} \sqrt{\left(-\frac{\mathrm{d} \ln T_{\mathrm{i}}}{\mathrm{d} r}+\frac{2}{3} \frac{\mathrm{d} \ln n_{\mathrm{e}}}{\mathrm{d} r}\right)\left(Z_{\mathrm{eff}} R\right)^{-1}-\frac{1}{8}\left(\frac{\mathrm{d} \ln n_{\mathrm{e}}}{\mathrm{d} r}+\frac{2}{R}\right)^{2}}$

$\gamma_{\max }^{\mathrm{DTE}} \approx f_{\mathrm{tr}} \eta_{\mathrm{e}} \frac{\omega_{*}^{2}}{v_{\mathrm{e}, \mathrm{eff}}}$

where $k_{\perp, \max }^{\mathrm{ITG}} \approx 0.3 / \rho_{\mathrm{s}}, k_{\perp, \max }^{\mathrm{DTE}} \approx 1 / \rho_{\mathrm{s}}$ with $\rho_{\mathrm{s}}$ being the ion Larmor radius,

$\eta_{\mathrm{e}}=\frac{\mathrm{d} \ln n_{\mathrm{e}}}{\mathrm{d} \ln T_{\mathrm{e}}}, \quad \nu_{\mathrm{e}, \mathrm{eff}}=\frac{1}{\tau_{\mathrm{ei}}} \frac{R}{r} \quad$ and $\quad \omega_{*}=\frac{c T_{\mathrm{e}} k_{\perp, \max }}{e B}\left(-\frac{\mathrm{d} \ln n_{\mathrm{e}}}{\mathrm{d} r}\right)$.

The edge transport is characterized by the coefficient (Endler et al 1995)

$$
\chi_{\perp}^{\mathrm{EDGE}} \approx \frac{c T_{\mathrm{e}}^{\mathrm{LCMS}}}{e B} \frac{\rho_{\mathrm{s}}^{\mathrm{LCMS}}}{L_{\mathrm{P}}^{\mathrm{LCMS}}}
$$


where $L_{\mathrm{P}}$ is the e-folding length of the electron pressure and the superscript LCMS denotes that the parameters are computed at the last closed magnetic surface. To simulate the decrease towards the plasma core of the anomalous transport triggered by the turbulence in the SOL, an empirical factor $\exp \left(-\left(r_{\mathrm{LCMS}}-r / L_{\mathrm{P}}\right)\right)$ has been introduced.

The electron diffusivity is adopted in the form

$$
D_{\perp}=\chi_{\perp}^{\mathrm{ITG}} f_{\mathrm{tr}}+\chi_{\perp}^{\mathrm{DTE}}+\chi_{\perp}^{\mathrm{EDGE}} .
$$

This takes into account that the main contribution of ITG turbulence to the particle transport comes from stochastization of the orbits of trapped electrons and should therefore be proportional to their relative abundance $f_{\text {tr }}$ (Horton et al 1985). The latter is determined by the inhomogeneity of the toroidal magnetic field and by the efficiency of detrapping through Coulomb collisions

$$
f_{\mathrm{tr}}=\sqrt{\frac{2 r}{r+R}} \exp \left[-\sqrt{\frac{q R}{\lambda_{\mathrm{e}}}\left(\frac{R}{r}\right)^{3 / 2}}\right] .
$$

The anomalous particle pinch velocity is governed mainly by the magnetic shear (Isichenko et al 1995, Yankov and Nycander 1997, Baker and Rosenbluth 1998)

$$
V_{\perp}=\left(\chi_{\perp}^{\mathrm{ITG}} f_{\mathrm{tr}} \varepsilon+\chi_{\perp}^{\mathrm{DTE}}\right) \frac{\mathrm{d} \ln q}{\mathrm{~d} r} .
$$

The small factor $\varepsilon=(4 r / 3 R)$ reflects a relatively weak effect on convection of instabilities caused by ion dynamics, in particular, ITG instability (Isichenko et al 1995, Baker and Rosenbluth 1998). The particle transport due to the instabilities under consideration is ambipolar since it results from drifts due to fluctuating electric fields. This implies that the diffusivities of electrons and ions are close to each other. For the pinch velocity of impurity particles we adopt a neoclassical one, which is caused by the density and temperature gradients of the background ions (Wenzel and Sigmar 1990)

$$
\begin{aligned}
& D_{\perp}^{Z}=D_{\perp} \\
& V_{\perp}^{Z}=V_{\perp}^{Z, \mathrm{NEO}} .
\end{aligned}
$$

In the heat conduction coefficients,

$$
\begin{aligned}
\kappa_{\perp}^{\mathrm{i}} & =\kappa_{\perp}^{\mathrm{i}, \mathrm{NEO}}+\left(\chi_{\perp}^{\mathrm{ITG}}+\chi_{\perp}^{\mathrm{EDGE}}\right) n_{\Sigma} \\
\kappa_{\perp}^{\mathrm{e}} & =\left(\chi_{\perp}^{\mathrm{ITG}} f_{\mathrm{tr}}+\chi_{\perp}^{\mathrm{DTE}}+\chi_{\perp}^{\mathrm{EDGE}}\right) n_{\mathrm{e}}
\end{aligned}
$$

the neoclassical contribution is computed by taking into account all ion species (Wesson 1997).

It is important to note that the present model for the suppression of turbulence is based on the interplay between diffusive and convective components of the particle flows. In the edge and internal barriers, the reduction in the transport is attributed normally to sheared $\Omega_{E \times B}$ rotation (Staebler 1998). Our calculations show (Tokar et al 2000) that this mechanism does not dominate in a transition to the RI-mode, when transport improves in a significant part of the plasma volume and profiles are not extremely steep anywhere.

\section{Results of modelling}

\subsection{Influence of gas puff intensity on plasma profiles}

The 1D transport code RITM with the anomalous transport model described above has been used to simulate the confinement rollover from the RI-mode to the L-mode in plasmas with a strong gas puff. The calculations have been arranged in order to reproduce the experimental 


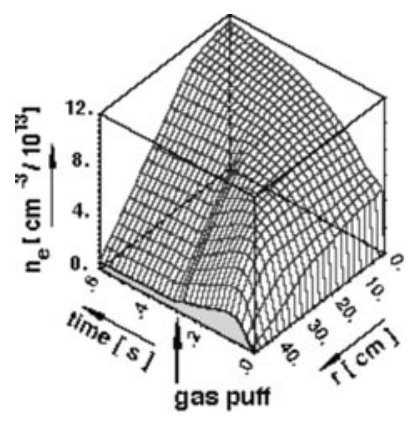

(a)

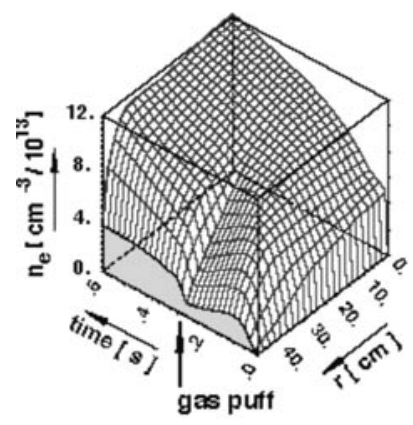

(b)

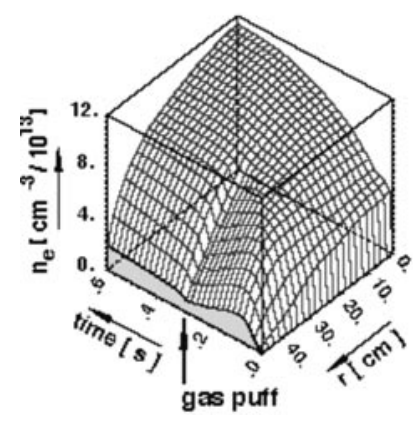

(c)

Figure 2. Influence of the fuelling rate and the edge diffusion coefficient on the evolution of density profile: (a) slow gas puff, $f_{\text {gas }}(R I), D_{\text {edge }}(R I) ;(b) f_{\text {gas }}=f_{\text {gas }}(R I) \times 3, D_{\text {edge }}=D_{\text {edge }}(R I)$; (c) $f_{\text {gas }}=f_{\text {gas }}(R I) \times 4, D_{\text {edge }}=D_{\text {edge }}(R I) \times 3$.

density profiles and also to obtain $Z_{\text {eff }}$ in accordance with the experimental value. The computations are carried out with a constant neon puff, starting from the beginning of the calculations, and with the ion temperature profile evolution taken from the experiment. Figure 2 shows the time evolution of the electron density profile modelled with various gas puffs. The calculations start from the same initial parabolic profiles. Then the gas influx is feedback controlled by the prescribed program for the ramp of the line-averaged density. It is important to notice that, due to the presence of inward convection in the electron flow, any skin-like behaviour of the density profile is absent during the whole time of the calculations. This is in agreement with experimental observations. At a time of $0.3 \mathrm{~s}$ quasi-stationary RI-mode conditions are reached, which are characterized by a peaked density profile, a high radiation fraction $P_{\text {rad }} / P_{\text {tot }} \sim 0.7$ and by ITG mode suppression over a wide radial zone of the plasma. Figures $2(a), 2(b)$ and $2(c)$ correspond to different intensities of the gas puff maintained constant from this time until the end of the calculations at $t=0.6 \mathrm{~s}$.

The density profile with a slow gas puff into the RI-mode, presented in figure $2(a)$, continues to peak and the central electron density exceeds $1.1 \times 10^{14} \mathrm{~cm}^{-3}$. During the whole time, the effective ion charge, $Z_{\text {eff }}$ (figure 3, curve a), is in the range of 1.8-2.4. The simulated evolution of the profiles is typical of that experimentally observed during the transition from the L-mode to the RI-mode. The density peaking and the increasing $Z_{\text {eff }}$ are instrumental in bringing about this transition, as follows from the following qualitative consideration. An increase of $Z_{\text {eff }}$ with a puff of impurities causes a reduction of the transport governed by the ITG instability, as is apparent from equation (8). The total electron flux prescribed by equation (4), including the contributions from ITG and DTE modes, changes so that the diffusive component of flow governed mainly by ITG turbulence decreases with respect to the convective part. The prevalence of the convective flux leads to the peaking of the density and to the further suppression of ITG turbulence mainly through the last term in $\gamma^{\text {ITG }}$ being nonlinear with respect to the density gradient. With time, this combination of density peaking and turbulence suppression penetrates deeper into the plasma, and the final state is characterized by an extremely peaked density and by ITG turbulence suppression in a wide plasma region. Simultaneously, with the increase of the line-averaged density, the edge density is maintained at a relatively low level in agreement with experimental observations (see figure 1). In the discharge shown in figure 2(b) the gas puff is increased at $t=0.3 \mathrm{~s}$ by a factor of three with respect to the level assumed in the previous case. This results in a strong broadening of the density profile in rough agreement with the experimental behaviour. 


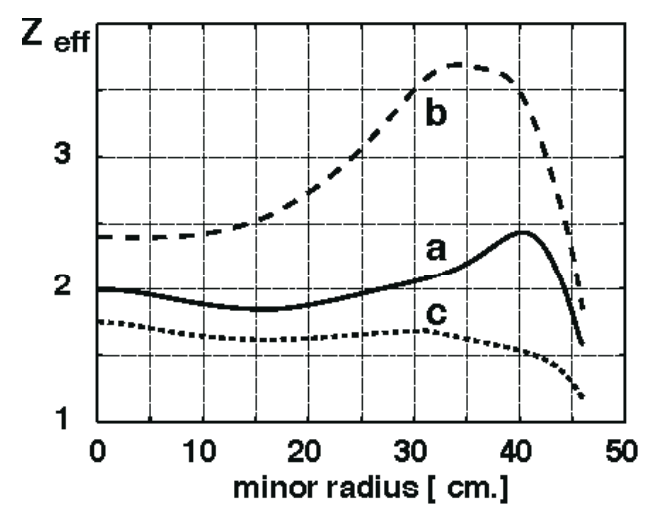

Figure 3. $Z_{\text {eff }}$ profiles for the discharges pertaining to figure 2: curves $\mathrm{a}, \mathrm{b}$ and $\mathrm{c}$ correspond to figures 2(a), 2(b) and 2(c), respectively.

However, the increase in the edge density and its gradient significantly exceed the measured values and the density profile is too flat in the outer plasma region. Moreover, the increase of the gas puff results in a further rise of $Z_{\text {eff }}$ in the whole plasma column with respect to the level in the RI-mode (figure 3, curve b). This disagrees with experiment, which shows a significant decrease of $Z_{\text {eff }}$ with the intense gas puff.

Thus, the computations show that the effect of a strong gas puff cannot be simulated by an increase of neutral particle influx alone but that, additionally, the transport of charged particles should be increased. In the plasma core this occurs automatically because the broadening of the density profile leads to a restart of the ITG-induced transport. However, the charged particles cannot leave the plasma since their diffusivity at the plasma edge is limited by the present edge transport model given by equation (10). Moreover, $\chi_{\perp}^{\text {EDGE }}$ even decreases when the temperature at the LCMS drops with the neutral puff. Figure $2(c)$ shows the density profile computed under the assumption that, simultaneously with the intensification of the gas puff at $t=0.3 \mathrm{~s}$, the edge diffusion coefficient is increased by a factor of four with respect to the level prescribed by equation (10). In this case, the flattening of the density profile occurs in agreement with the experimental observations without the formation of an extremely high density and density gradient at the LCMS. Simultaneously, the high edge diffusion allows more impurity ions to escape from the plasma and the $Z_{\text {eff }}$ value reduces in the whole plasma (see figure 3, curve c).

The steady-state profiles finally reached in these simulated discharges are quite different (see figure 4). The calculated density profile in the case of a slow gas puff (figure 4(a), curve 1) reproduces well the experimental RI-mode density profile (figure 4(a), curve 3 ) and has a steep gradient over a wide plasma zone. As a result, the ITG mode is suppressed and the diffusion flow caused by this turbulence is reduced over two thirds of the radius (figure $4(b)$, curve 1). Curve 2 in figure 4(a) shows the density profile for the modelled discharge in which the gas puff is increased by a factor of four and the edge diffusion coefficient is increased by a factor of three with respect to the first simulation. This profile also mimics the shape of the experimental density profile after the RI-L back transition caused by a strong gas puff (figure 4(a), curve 4). Nevertheless there is a difference of $20 \%$ in the line-averaged densities between experimental and RITM-simulated density profiles. This discrepancy arises from the different values of the density at the LCMS, where the simulation assumes as boundary conditions fixed e-folding lengths for the densities and temperatures. We expect that a more 


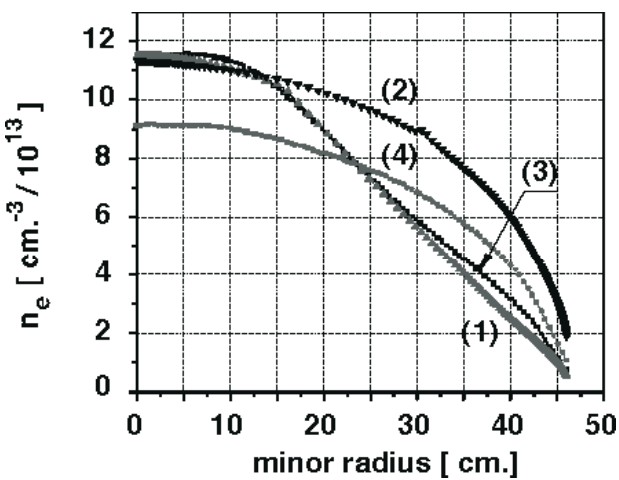

(a)

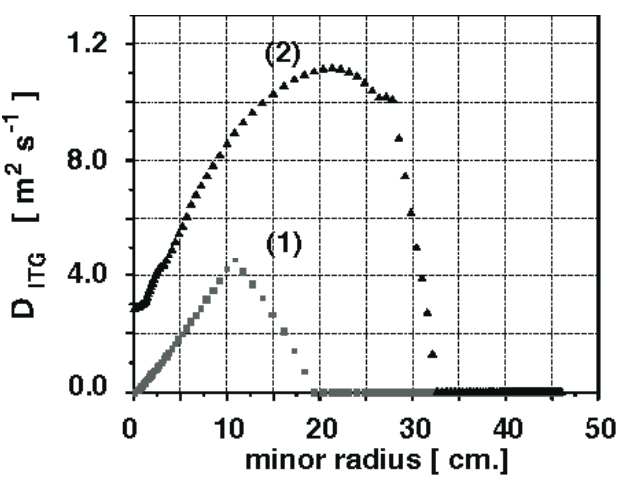

(b)

Figure 4. Profiles of the electron density $(a)$ and the ITG-induced diffusion coefficient $(b)$ for the discharges shown in figures 2(a) and 2(c) (curves 1 and 2 respectively) and the experimental counterparts in the RI- and L-modes (curves 3 and 4 respectively).

sophisticated model for the SOL, taking into account ionization of neutrals in the SOL, would improve the numerical results.

\subsection{RI-L bifurcation caused by a strong gas puff}

In earlier work (Tokar et al 2000), the transition from the L-mode to the RI-mode caused by seeding of impurities has been interpreted as a bifurcation made possible by the particular parametric dependence of the electron flow upon the peaking factor $p=\mathrm{d} \ln n_{\mathrm{i}} / \mathrm{d} \ln T_{\mathrm{i}}$ (the inverse value of the parameter $\eta_{\mathrm{i}}$ often used in ITG models). When plotting the electron flow versus $p$, an $\mathrm{N}$-shaped dependence is obtained (see figure 5). Here we intend to show that the same model is capable of explaining how a strong gas puff can provoke a back bifurcation from the RI-mode to the L-mode.

The value of the peaking factor at a given radial position can be determined from the integral of the continuity equation for electrons (equation (2))

$$
\begin{gathered}
n_{\mathrm{e}}\left[\left(\frac{p}{L_{T_{\mathrm{i}}}}-\frac{4 r}{3 R L_{q}}\right) f_{\mathrm{tr}} \chi_{\mathrm{ITG}}(p)+\left(\frac{p}{L_{T_{\mathrm{i}}}}-\frac{1}{L_{q}}\right) \chi_{\mathrm{DTE}}(p)+\frac{p}{L_{T_{\mathrm{i}}}} \chi_{\mathrm{EDGE}}\right] \\
-\frac{1}{r} \int_{0}^{r}\left(S_{\mathrm{e}}-\frac{\partial n_{\mathrm{e}}}{\partial t}\right) r \mathrm{~d} r \equiv G(p)=0
\end{gathered}
$$

where $L_{T_{\mathrm{i}}}=-\left(\mathrm{d} r / \mathrm{d} \ln T_{\mathrm{i}}\right), L_{q}=-(\mathrm{d} r / \mathrm{d} \ln q)$ and $S_{\mathrm{e}}$ is the electron source term.

Figure 5 shows the function $G(p)$ calculated for the conditions pertaining to four time instants in figure $2(c)$ at the minor radius $30 \mathrm{~cm}$ where the variation in the density gradients during the stage of intensive gas puff is most pronounced. The value of the peaking factor at a given time corresponds to the points where the function $G(p)$ reduces to zero. The number of such states is determined by the position of the maximum and minimum values of $G(p), G^{\max }$ and $G^{\mathrm{min}}$, with respect to zero level. In the case of curve 1, computed for the L-mode state at $t=0.23 \mathrm{~s}, G^{\min } \leqslant 0 \leqslant G^{\max }$ and there are three possible solutions. For $t<0.23 \mathrm{~s}$, there is no density peaking and the solution (A) at the minimal $p$ is realized in the RITM calculations. Due to the increase of $Z_{\text {eff }}$ caused by neon seeding, $G^{\text {max }}$ decreases because of the $Z_{\text {eff }}$ dependence in $\gamma^{\text {ITG }}$. When $G^{\text {max }}$ becomes negative only a single solution (B) remains, in which the density peaking is high. This is demonstrated by curve 2 for the RI-mode at $t=0.29 \mathrm{~s}$. A strong gas 


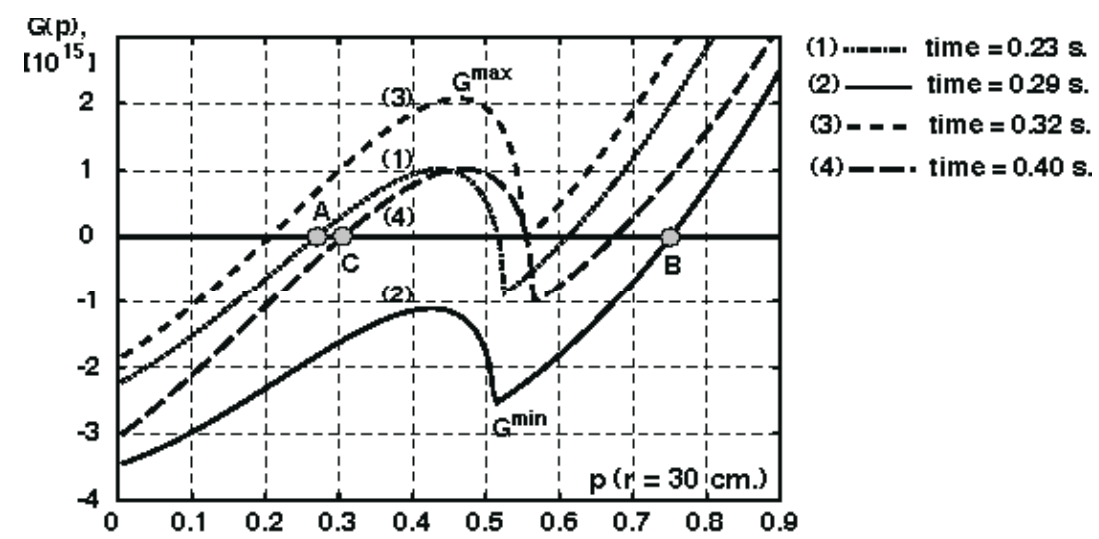

Figure 5. The $G$ function versus the peaking factor $p$ for four time instants in the modelled discharge for the conditions pertaining at $r=30 \mathrm{~cm}$.

puff beginning from $t=0.3 \mathrm{~s}$ results in an upwards shift of $G(p)$ on account of increasing local density, positive $\partial n_{\mathrm{e}} / \partial t$ moves $G^{\mathrm{min}}$ upwards, and $G^{\mathrm{max}}$ increases with decreasing $Z_{\mathrm{eff}}$. When $G^{\mathrm{min}}$ approaches zero (curve 3 ) the plasma switches into a state on the branch where $\gamma^{\text {ITG }}>0$. As $\partial n_{\mathrm{e}} / \partial t$ eventually diminishes with the attainment of steady-state conditions and $G^{\mathrm{min}}$ returns below zero, $G^{\mathrm{max}}$ still remains positive because $Z_{\text {eff }}$ decreases due to loss of impurities with increased edge transport. Finally, the plasma settles into the stable stationary state (C) on curve 4, corresponding to the L-mode.

\section{Experimental confirmation of the increase in edge transport with a strong gas puff}

Figure 6 shows the frequency spectra of the density fluctuation measured by a microwave reflectometer during the discharges of figure 1 (Dreval et al 1999). The position of the reflection layer estimated from the cut-off density, corresponding to the reflectometer frequency, is on the top and 2-3 cm inside the LCMS towards the plasma core. It is seen that both discharges start from the same frequency spectrum of fluctuations. This spectrum remains unchanged in the discharge with a slow gas puff. In contrast, the intense gas puff leads to a sharp increase of the fluctuation amplitude in the frequency range below $50 \mathrm{kHz}$ (Unterberg et al 2000). The shift of the centre of the initial spectrum $(t=1.3 \mathrm{~s})$ is not discussed in this paper and could be due to a plasma rotation which would cause a Doppler shift of the measured frequencies (Crowley and Mazzucato 1985). It should be noted that the same changes in the fluctuation spectrum were noticed in other discharges where the confinement degradation was observed to take place with the same moderate gas puff but under not optimized wall conditions.

Figure 7 shows the correlation between the plasma edge parameters and the width of the auto-correlation function, $\Delta_{\mathrm{ACF}}$, relating to the wavelengths of fluctuations. The whole data set is taken at a given magnetic field $B_{\mathrm{t}}=2.24 \mathrm{~T}$ and plasma current $I_{\mathrm{P}}=380 \mathrm{kA}$. The areas corresponding to the RI- and L-modes are separated by the grey curve where the energy confinement time, $\tau$, is $80 \%$ of that predicted by the RI-mode scaling $\tau_{\mathrm{RI}} \approx k \bar{n}_{\mathrm{e} 0}\left(P_{\mathrm{tot}}\right)^{-2 / 3}$, where $k \approx 0.18, \bar{n}_{\mathrm{e} 0}$ is the central line-averaged density in $10^{20} \mathrm{~m}^{-3}$, and $P_{\text {tot }}$ is the total input power in MW (Weynants et al 1999, Messiaen et al 1999). It is seen that $\Delta_{\mathrm{ACF}}$ increases when the plasma edge parameters cross the grey curve going from the RI-mode to the L-mode as a result of an intense gas puff. If one assumes that the plasma rotation does not change significantly in the considered sequence of discharges at the radii near the cut-off layer, then the 

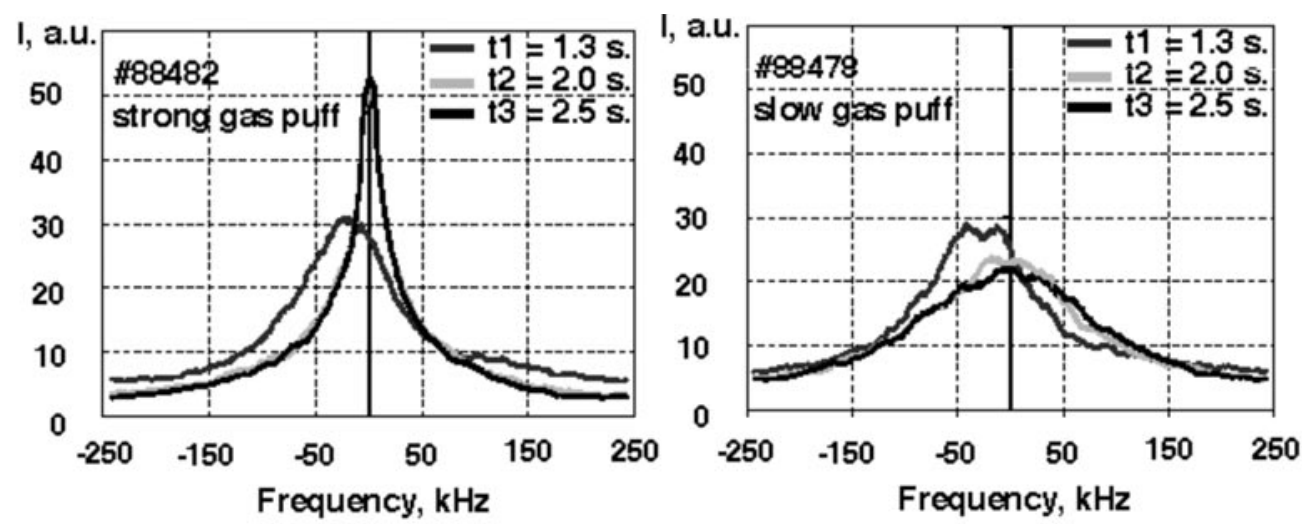

Figure 6. Frequency spectra of density fluctuations measured by the microwave reflectometer from $2-3 \mathrm{~cm}$ inside the LCMS

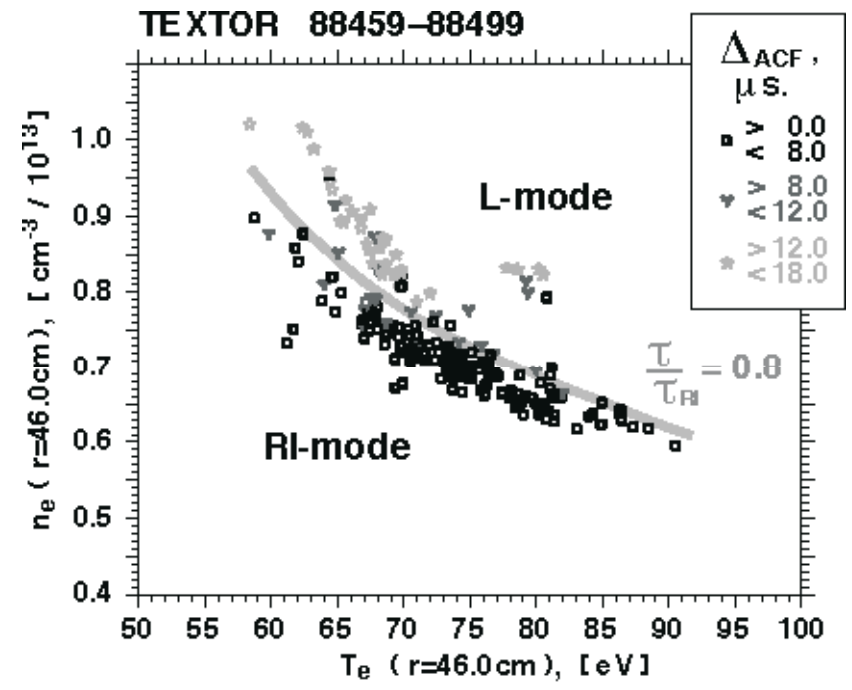

Figure 7. Variation of the auto-correlation function, $\Delta_{\mathrm{ACF}}$, measured by reflectometer $(r \sim 44 \mathrm{~cm})$, with the plasma edge parameters and its correlation with the confinement performance.

$\Delta_{\mathrm{ACF}} \sim\langle\lambda\rangle \sim 1 /\left\langle k_{\perp}\right\rangle$, where $\lambda$ is the wavelength of turbulence, averaged over the frequencies and the reflecting volume, and $k_{\perp}$ is the poloidal component of the wave vector. As the diffusion coefficient is proportional to $1 / k_{\perp}^{2}$ (Kadomtsev and Pogutse 1971), figure 7 therefore suggests, in agreement with the RITM modelling, that anomalous transport in the plasma edge increases at the time of the confinement deterioration. Additionally, this increase of the edge particle transport is confirmed by the drop in the particle confinement time observed during the intense gas puff (see figure 1).

\section{Discussion of results}

The cause of the increase in the edge transport with intense gas puffing is not completely clear at the moment. One possible line of thinking could be to invoke the action of neutrals on 
anomalous edge transport, for which several mechanisms can be found in the literature.

One such possibility would be amplification of the drift resistive ballooning (DRB) instability as a result of the gas puff. In figure 1, it is seen that a slow gas puff keeps $n_{\mathrm{e}}$ LCMS low and energy confinement high, whereas the strong gas puff in the shot 88482 (solid curves) leads to a noticeable increase of $n_{\mathrm{e}}$ LCMS and a simultaneous reduction of the edge temperature (not shown). The latter modifications result in an increase of the electron collisional frequency $\nu_{\mathrm{e}}$ and lead to an increase of the transport due to DRB unstable modes (Gusdar et al 1993): $D_{\mathrm{DRB}} \approx\left(2 \pi q \rho_{\mathrm{e}}\right)^{2} v_{\mathrm{e}}\left(R / L_{n}\right)$. An estimate gives a value of $1-2 \mathrm{~m}^{2} \mathrm{~s}^{-1}$, which is smaller than a necessary level of 3-5 $\mathrm{m}^{2} \mathrm{~s}^{-1}$.

Besides, or in addition to, this effect neutrals could be responsible for enhancing drift wave instabilities, through their ionization and friction with ions in charge-exchange interactions. This channel could be important since the measurements reveal a strong increase of the neutral pressure at the edge, $P_{\text {neu }}$, in obvious correlation with confinement degradation (see figure 1).

As was demonstrated by Linson and Workman (1970) the friction of plasma ions with neutrals due to charge-exchange processes in the ionosphere can lead, under specific conditions, to the excitation of new drift instabilities. Recently, this idea has been applied to explain anomalous transport in the SOL plasma of divertor tokamaks by Daughton et al (1998). The underlying mechanism can be understood from figure 8 , in which a drift wave perturbation of the plasma density is considered, $\tilde{n} \propto \exp \left(-\mathrm{i} \omega t+\mathrm{i} k_{y} y+\mathrm{i} k_{z} z\right)$, where $k_{y, z}$ are components of the wave vector. In the presence of a radial flow of neutrals, the friction force on the ions generates a poloidal charged particle drift, leading to a perturbation of the poloidal current $\tilde{j}_{y}$

$$
\tilde{F}_{\mathrm{fr}}=\frac{1}{c m_{\mathrm{i}}}\left[\tilde{j}_{y} B_{0}\right] \text {. }
$$

Since the total current perturbation is divergence free, a distortion of the current component parallel to the magnetic field arises: $\tilde{j}_{z}=-\tilde{j}_{y} k_{y} / k_{z}$. As a result, the Boltzmann distribution of the electric potential of the wave is broken in such a way that the radial drift caused by the poloidal electric field can lead to the growth of the wave amplitude.

Daughton et al (1998) have demonstrated that, for an instability to grow, the gradient of neutral pressure should have the same direction as the gradient of the unperturbed plasma density. Such a situation can be realized in the vicinity of a strong neutral source such as, for example, produced by strong gas puffing. Here a total number of neutrals launched into the plasma is comparable with the total loss of plasma particles through the LCMS. The ionization

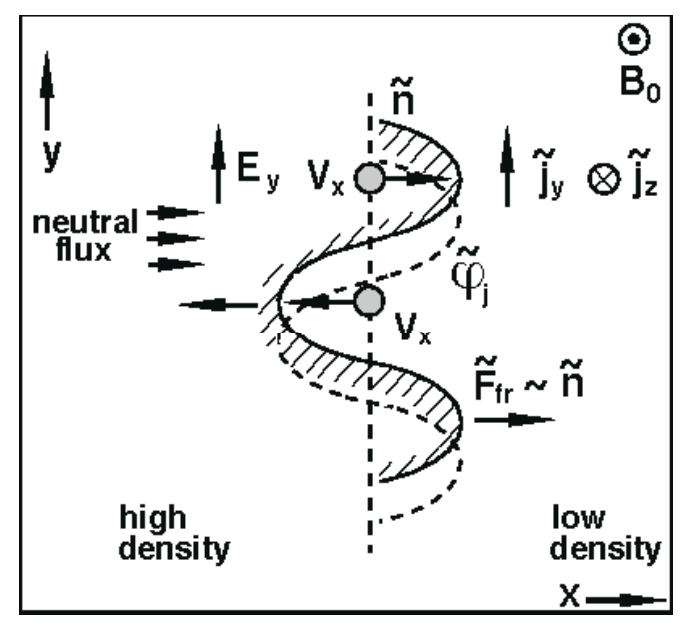

Figure 8. Destabilization of drift waves by friction with neutrals. 
of these neutrals can provide a local maximum in the density of charged particles (Tokar 1993) so that the resulting radial gradient of the ion density can have the sign required for the instability.

No firm appraisal of this mechanism can be made without further and complex modelling. However, in the limit of a very large influx of neutrals, the growth rate of the instability can be roughly estimated (Daughton et al 1998) as $\gamma \approx \sqrt{\left(k_{\mathrm{cx}} j_{a}\right) / L_{n}}$, where $k_{\mathrm{cx}} \approx 3 \times 10^{-8} \mathrm{~cm}^{3} \mathrm{~s}^{-1}$ is the charge-exchange rate coefficient, $j_{a}$ is the density of neutral flux and $L_{n}$ is the plasma density e-folding length. By taking $j_{a}$ as the averaged over the magnetic surface density of a local influx of $10^{22}$ neutrals and $L_{n} \approx 1 \mathrm{~cm}$, one obtains $\gamma \approx 3 \times 10^{4} \mathrm{~s}^{-1}$. For $k_{y} \geqslant 1 \mathrm{~cm}^{-1}$ (for perturbations of smaller $k_{y}$ the distortion of parallel current is too small), a mixing length approximation results in an increase of the particle diffusivity by some $\mathrm{m}^{2} \mathrm{~s}^{-1}$. The above consideration does not take into account the contribution to the plasma density perturbations from the ionization of neutrals. This can lead to the so-called ionization instability analysed by several authors (see, for example, Ware et al 1992) and can additionally increase the effect of neutrals on anomalous transport.

The total effect from instabilities caused by direct influence of neutrals on the plasma motion and from DRB modes could explain the increase of the edge transport needed for an interpretation of our findings.

Finally, note that the changes at the plasma edge caused by the gas puff include a moderate modification of the plasma rotation. Therefore, in a more sophisticated model of the edge transport which is under development, the effect of rotation on drift modes will be taken into account.

\section{Conclusion}

By the optimization of the gas puff rate and wall conditions it is possible to achieve H-mode quality confinement in the RI-mode at densities well above the Greenwald density limit. Too strong a gas puff leads, however, to confinement degradation towards the L-mode, apparently as the result of a significantly increased edge transport. The latter follows from the results of RITM-code modelling and is supported by changes in the density fluctuation spectrum measured by reflectometer. The loss of puffed impurities with increased edge transport results in a decrease of $Z_{\text {eff }}$. Both this and the flattening of the density profile caused by the gas puff are crucial factors for the restart of ITG turbulence in a wide plasma zone with accompanying confinement degradation.

\section{Acknowledgments}

The TEXTOR-94 team is gratefully acknowledged for providing excellent experimental conditions. We wish to thank M Lehnen for providing valuable experimental data. This work has been carried out as part of a PhD thesis supported by Graduiertenkolleg of Heinrich Heine University Duesseldorf.

\section{References}

Baelmans et al 1995 Proc. 22nd European Physical Society Conf. on Controlled Fusion and Plasma Physics vol 19C, ch IV, p 321

Baker D R and Rosenbluth M N 1998 Phys. Plasmas 52936

Crowley T and Mazzucato E 1985 Nucl. Fusion 25507

Daughton W et al 1998 Phys. Plasmas 52217 
Dreval V et al 1999 Proc. 26th European Physical Society Conf. on Controlled Fusion and Plasma Physics vol 23J, p 801

Endler M et al 1995 J. Nucl. Mater. 220-2 293

Greenwald M et al 1988 Nucl. Fusion 282199

Gusdar P N et al 1993 Phys. Fluids B 53712

Hirshman S P et al 1977 Nucl. Fusion 17611

Horton L D et al 1999 Nucl. Fusion 391

Horton W et al 1985 Plasma Phys. Control. Fusion 27937

Isichenko M B et al 1995 Phys. Rev. Lett. 744436

Kadomtsev B B and Pogutse O P 1971 Nucl. Fusion 1167

Linson L M and Workman J B 1970 J. Geophys. Res. 753211

Mank G et al 2000 Phys. Rev. Lett. 852312

Messiaen A et al 1999 Proc. 26th European Physical Society Conf. on Controlled Fusion and Plasma Physics vol 23J, p 605

Nordman H et al 1990 Nucl. Fusion 33983

Petrie T W et al 1993 Nucl. Fusion 33929

Solov'ev L S and Shafranov V D 1970 Rev. Plasma Phys. 51

Staebler G M 1998 Plasma Phys. Control. Fusion 40569

Suttrop W et al 1999 J. Nucl. Mater. 266-9 118

Tokar M Z 1993 Plasma Phys. Control. Fusion 351119

_ 1994 Plasma Phys. Control. Fusion 361819

Tokar M Z et al 2000 Phys. Rev. Lett. 84895

Unterberg B et al 2000 Improved energy confinement at trans-Greenwald densities in discharges with a radiating edge in the tokamak TEXTOR-94, IAEA-CN-77/EX5/2

Ware A S et al 1992 Phys. Fluids B 4877

Wenzel K W and Sigmar D J 1990 Nucl. Fusion 301117

Wesson J 1997 Tokamaks (Oxford Engineering Science Series) vol 48, 2nd edn (Oxford: Clarendon)

Weynants R R et al 1999 Nucl. Fusion 391637

Yankov V V and Nycander J 1997 Phys. Plasmas 42907 